\title{
Ordered Valence-Bond States in Symmetric Two-Dimensional Spin-Orbital Systems
}

\author{
Guang-Ming Zhang ${ }^{1,2}$ and Shun-Qing Shen ${ }^{1}$ \\ ${ }^{1}$ Department of Physics, the University of Hong Kong, Pokfulam Road, Hong Kong, China \\ ${ }^{2}$ Center for Advanced Study, Tsinghua University, Beijing, 100084 China
}

(Received 25 May 2001; published 20 September 2001)

\begin{abstract}
We consider a superexchange Hamiltonian, $H=-\sum_{\langle i, j\rangle}\left(2 \mathbf{S}_{i} \cdot \mathbf{S}_{j}-\frac{1}{2}\right)\left(2 \mathbf{T}_{i} \cdot \mathbf{T}_{j}-\frac{1}{2}\right)$, which describes systems with orbital degeneracy and strong electron-phonon coupling in the limit of large on-site repulsion. In an $\mathrm{SU}(4)$ Schwinger boson representation, a reduced spin-orbital interaction is derived exactly, and a mean field theory has been developed. In one dimension, a spin-orbital liquid state with a finite gap is obtained. On a two-dimensional square lattice a novel type of spin-orbital ferromagnetically ordered state appears, while spin and orbital are antiferromagnetic. An important relation has been found, relating the spin and orbital correlation functions to the combined spin-orbital ones.
\end{abstract}

DOI: 10.1103/PhysRevLett.87.157201

There has been much interest in the properties of Mott insulators with orbital degeneracy [1-4], where the electron configuration has an orbital degeneracy in addition to the spin degeneracy. Because of the interplay between the spin and orbital degrees of freedom, a rich variety of spin and orbital ordering effects have been displayed [5-9], and a new combined spin-orbital degrees of freedom may introduce some new physics to the transitional metal oxides [10-15]. Recently, in order to describe the low-energy physics of an insulating crystal with one electron per site with double orbital degeneracy in the limit of the large on-site Hubbard repulsion, a superexchange Hamiltonian was proposed [16,17]:

$$
H=-\sum_{\langle i, j\rangle}\left(2 \mathbf{S}_{i} \cdot \mathbf{S}_{j}-\frac{1}{2}\right)\left(2 \mathbf{T}_{i} \cdot \mathbf{T}_{j}-\frac{1}{2}\right),
$$

where two isotropic Heisenberg antiferromagnets (AFM) are coupled by a quartic term on equivalent bonds, and $\mathbf{S}_{i}$ and $\mathbf{T}_{i}$ denote the spin-1/2 and orbital-1/2 operators at a lattice site $i$, respectively. The physical condition for deriving the above effective model Hamiltonian is that, among the possible two-particle states obtained upon virtual hopping, the interorbital singlet is the lowest energy state due to the dynamical Jahn-Teller effect. The condition might be realized in the large body of new molecular compounds based on $\mathrm{C}_{60}$ [18] or layered fullerides and some twodimensional (2D) copolymers [19]. Based on numerical calculations, Santoro et al. argued that the ground state of the model shows a spin-Peierls-like dimerization in one dimension (1D) [16], while on a 2D square lattice no evidence of order of any kind has been found so that the ground state is concluded to be a spin liquid of resonating valence bonds (VB) [17]. In order to put these results on firm ground, it is thus of great interest to develop new approaches to this model.

In this Letter, we use an SU(4) Schwinger boson representation [20] to denote both the spin- $1 / 2$ and orbital-1/2 operators, and the model Hamiltonian is simplified to a reduced interaction, describing a symmetric pairing attraction among the nearest-neighbor hard-core bosons. By in-
PACS numbers: 75.10.Jm, 11.10.Hi, 11.25.Hf, 75.40.Mg

troducing a symmetric VB order parameter, a mean field theory is developed. In 1D, a spin-orbital liquid state with a finite gap in the excitation spectra is obtained, corresponding to a quantum disordered VB state. On a 2D square lattice, due to the presence of the Bose-Einstein condensations, we found a novel spin-orbital ferromagnetically (FM) ordered state, corresponding to a short-ranged VB crystal state, while both the spin and orbital degrees of freedom form an AFM ordering.

The model Hamiltonian can be rewritten as follows:

$$
\begin{aligned}
H= & 2 \sum_{\langle i, j\rangle}\left(\mathbf{S}_{i} \cdot \mathbf{S}_{j}+\mathbf{T}_{i} \cdot \mathbf{T}_{j}\right) \\
& -\sum_{\langle i, j\rangle}\left(2 \mathbf{S}_{i} \cdot \mathbf{S}_{j}+\frac{1}{2}\right)\left(2 \mathbf{T}_{i} \cdot \mathbf{T}_{j}+\frac{1}{2}\right),
\end{aligned}
$$

where the direct quadratic couplings among spins and orbitals are antiferromagnetic, and the quartic coupling between spins and orbitals is ferromagnetic. The model Hamiltonian has an $\mathrm{SU}(2) \otimes S U(2)$ symmetry, representing rotational invariance in both spin and orbital spaces, and also interchange symmetry between the spins and orbitals. Moreover, one also notices that the total spin, orbital, and combined staggered spin-orbital operators are as follows:

$$
\begin{gathered}
S^{\alpha}=\sum_{j} S_{j}^{\alpha}, \quad T^{\alpha}=\sum_{j} T_{j}^{\alpha}, \\
L^{\alpha \beta}=\sum_{j} e^{i \mathbf{Q} \cdot R_{j}} 2 S_{j}^{\alpha} T_{j}^{\beta},
\end{gathered}
$$

where $\mathbf{Q}$ is the AFM reciprocal vector, which generates an SU(4) Lie algebra and also commutes with the model Hamiltonian. Therefore, Eq. (1) possesses the SU(4) symmetry [16]. For a state with the SU(4) symmetry, implying that the state is invariant under the rotation in the SU(4) space, we have an identity for the static correlation functions

$$
\left\langle S_{i}^{\alpha} S_{j}^{\alpha}\right\rangle=\left\langle T_{i}^{\beta} T_{j}^{\beta}\right\rangle=e^{i \mathbf{Q} \cdot\left(\mathbf{R}_{i}-\mathbf{R}_{j}\right)}\left\langle 4 S_{i}^{\alpha} T_{i}^{\beta} S_{j}^{\alpha} T_{j}^{\beta}\right\rangle,
$$

where $\langle\cdots\rangle$ represents the expectation value on the $\mathrm{SU}(4)$ 
symmetric state. The underlying physics for the identities is very clear: if the spin and orbital degrees of freedom are antiferromagnetically correlated, the combined spin-orbital correlation functions will be ferromagnetic. This property originates from the additional phase factor in the generators of $L^{\alpha \beta}$. It should be mentioned that another spin-orbital model Hamiltonian $H^{\prime}=\sum_{\langle i, j\rangle}\left(2 \mathbf{S}_{i} \cdot\right.$ $\left.\mathbf{S}_{j}+\frac{1}{2}\right)\left(2 \mathbf{T}_{i} \cdot \mathbf{T}_{j}+\frac{1}{2}\right)$, the natural generalization of the $\mathrm{SU}(2)$ Heisenberg spin model and exactly soluble in 1D, has also been used to understand some orbital properties of transition metal oxides [10-15]. However, although these two models have the same symmetry, the fifteen generators of the SU(4) symmetry group are not the same, and the identities of the correlation functions induced from this symmetry are also different. In this sense, the physics involved in these two models are independent.

As we know, the Schwinger boson mean field approach has been a successful theory in describing the low-energy excitations of the conventional FM and AFM Heisenberg spin models [20,21]. In particular, starting from shortranged VB order parameters, it can produce either quantum disordered or quantum long-range ordered magnetic states. In our model, when two sets of SU(2) Schwinger bosons are introduced to denote the spin- $1 / 2$ and orbital-1/2 operators separately, we have

$$
\begin{array}{r}
2 \mathbf{S}_{i} \cdot \mathbf{S}_{j}+\frac{1}{2}=\sum_{\alpha, \beta} a_{i, \alpha}^{\dagger} a_{i, \beta} a_{j \beta}^{\dagger} a_{j, \alpha}, \\
2 \mathbf{T}_{i} \cdot \mathbf{T}_{j}+\frac{1}{2}=\sum_{m, n} d_{i, m}^{\dagger} d_{i, n} d_{j, n}^{\dagger} d_{j, m},
\end{array}
$$

with local constraints $\sum_{\alpha} a_{i, \alpha}^{\dagger} a_{i, \alpha}=1$ and $\sum_{m} d_{i, m}^{\dagger} d_{i, m}=$ 1 , where the indices $\alpha, \beta$ and $m, n$ are taken with values 1 and 2 corresponding to eigenstates of $S^{z}= \pm 1 / 2$ and $T^{z}= \pm 1 / 2$, respectively. Both $a_{i, \alpha}$ and $d_{i, m}$ operators satisfy the boson commutation relations. To treat the quartic spin-orbital interaction term on an equal footing as the spin-spin and orbital-orbital interactions, the fact has been used that in the model Hamiltonian the Hilbert space on each lattice site consists of four basic states in terms of $\left|S^{z} ; T^{z}\right\rangle$ :

$$
\begin{array}{ll}
\left|+\frac{1}{2} ;+\frac{1}{2}\right\rangle=|1\rangle, \quad\left|-\frac{1}{2} ;+\frac{1}{2}\right\rangle=|2\rangle, \\
\left|+\frac{1}{2} ;-\frac{1}{2}\right\rangle=|3\rangle, \quad\left|-\frac{1}{2} ;-\frac{1}{2}\right\rangle=|4\rangle .
\end{array}
$$

These four states form a set of local basis to represent the SU(4) symmetry group. The conventional SU(4) generators $J_{\mu}^{\nu}(i)$ act on a basic state $|\eta\rangle_{i}$ according to the equation $J_{\mu}^{\nu}(i)|\eta\rangle_{i}=\delta_{\nu, \eta}|\mu\rangle_{i}$ with a local constraint $\sum_{\mu} J_{\mu}^{\mu}(i)=1$, where the indices $\mu$ and $\nu$ are taken with values from 1 to 4 corresponding to the four eigenstates of $\left| \pm \frac{1}{2} ; \pm \frac{1}{2}\right\rangle$. The SU(4) Lie algebra is defined by the commutation relation

$$
\left[J_{m}^{n}(i), J_{k}^{l}(i)\right]=\delta_{n, k} J_{m}^{l}(i)-\delta_{m, l} J_{k}^{n}(i) .
$$

In terms of four-component hard-core bosons, we have $J_{\mu}^{\nu}(i)=b_{i, \mu}^{\dagger} b_{i, \nu}$ with the local constraint $\sum_{\mu} b_{i, \mu}^{\dagger} b_{i, \mu}=$ 1. Therefore, the quartic spin-orbital exchange interaction can be written as

$$
\begin{aligned}
\left(2 \mathbf{S}_{i} \cdot \mathbf{S}_{j}+\frac{1}{2}\right)\left(2 \mathbf{T}_{i} \cdot \mathbf{T}_{j}\right. & \left.+\frac{1}{2}\right) \\
& =\sum_{\mu, \nu} b_{i, \mu}^{\dagger} b_{i, \nu} b_{j, \nu}^{\dagger} b_{j, \mu} .
\end{aligned}
$$

By a projection procedure, both the spin-spin and orbitalorbital quadratic interactions can also be expressed in terms of the four-component hard-core bosons. Fortunately, most of the resulting terms are found to be exactly canceled with the quartic spin-orbital exchange interaction, and the resulting Hamiltonian is reduced to a simple and compact form,

$$
\begin{aligned}
H= & -\sum_{\langle i, j\rangle}\left[\left(b_{i, 1}^{\dagger} b_{j, 4}^{\dagger}+b_{i, 4}^{\dagger} b_{j, 1}^{\dagger}\right)-\left(b_{i, 2}^{\dagger} b_{j, 3}^{\dagger}+b_{i, 3}^{\dagger} b_{j, 2}^{\dagger}\right)\right] \\
& \times\left[\left(b_{j, 4} b_{i, 1}+b_{j, 1} b_{i, 4}\right)-\left(b_{j, 3} b_{i, 2}+b_{j, 2} b_{i, 3}\right)\right] .
\end{aligned}
$$

Actually, this reduced model Hamiltonian has explicitly displayed that the main physics of the symmetrically coupled spin-orbital interaction is to induce a unique attractive pairing instability among the nearest-neighbor hard-core bosons. We also emphasize here that the quartic spinorbital exchange interaction plays the same role as that of the spin-spin and orbital-orbital superexchange terms.

In the reduced spin-orbital model Hamiltonian, the local attractive interaction shares closely a resemblance to the effective model Hamiltonian in the conventional BCS superconductivity theory. Such a feature allows us to introduce an SU(4) short-ranged VB pairing order parameter

$$
\Delta=-\left\langle\left(b_{j, 4} b_{i, 1}+b_{j, 1} b_{i, 4}\right)-\left(b_{j, 3} b_{i, 2}+b_{j, 2} b_{i, 3}\right)\right\rangle,
$$

which one can assume to be real. A following mean field theory develops naturally, leading to the mean field Hamiltonian,

$$
\begin{aligned}
H_{\mathrm{mf}}= & \lambda \sum_{i, \mu} b_{i, \mu}^{\dagger} b_{i \mu}-\lambda N+Z \Delta^{2} N \\
& +\Delta \sum_{\langle i, j\rangle}\left[\left(b_{i, 1}^{\dagger} b_{j, 4}^{\dagger}+b_{i, 4}^{\dagger} b_{j, 1}^{\dagger}\right)\right. \\
& \left.-\left(b_{i, 2}^{\dagger} b_{j, 3}^{\dagger}+b_{i, 3}^{\dagger} b_{j, 2}^{\dagger}\right)+\text { H.c. }\right],
\end{aligned}
$$

where the local constraint has been imposed on average through a Lagrangian multiplier, $N$ is the total number of lattice sites, and $Z$ is the number of the nearest neighbors. In the momentum space, $H_{\mathrm{mf}}$ becomes

$$
\begin{aligned}
H_{\mathrm{mf}}= & \lambda \sum_{\mathbf{k}, \mu} b_{\mathbf{k}, \mu}^{\dagger} b_{\mathbf{k}, \mu}-\lambda N+Z \Delta^{2} N \\
& +2 Z \Delta \sum_{\mathbf{k}} \gamma_{\mathbf{k}}\left[\left(b_{\mathbf{k}, 1}^{\dagger} b_{-\mathbf{k}, 4}^{\dagger}-b_{\mathbf{k}, 2}^{\dagger} b_{-\mathbf{k}, 3}^{\dagger}\right)+\text { H.c. }\right],
\end{aligned}
$$

where $\gamma_{\mathbf{k}}=\frac{1}{Z} \sum_{\delta} e^{i \mathbf{k} \cdot \delta}$ and the vector $\delta$ points to the nearest-neighbor sites. When a Nambu spinor is defined by 
$\Psi^{\dagger}(\mathbf{k})=\left(b_{\mathbf{k}, 1}^{\dagger}, b_{\mathbf{k}, 2}^{\dagger}, b_{\mathbf{k}, 3}^{\dagger}, b_{\mathbf{k}, 4}^{\dagger} ; b_{-\mathbf{k}, 4}, b_{-\mathbf{k}, 3}, b_{-\mathbf{k}, 2}, b_{-\mathbf{k}, 1}\right)$, the mean field Hamiltonian is expressed in the compact matrix form

$$
H_{\mathrm{mf}}=\frac{1}{2} \sum_{\mathbf{k}} \Psi^{\dagger}(\mathbf{k}) H_{\mathrm{mf}}(\mathbf{k}) \Psi(\mathbf{k})+Z \Delta^{2} N-3 \lambda N,
$$

with $H_{\mathrm{mf}}(\mathbf{k})=\lambda \Omega_{0}+2 Z \Delta \gamma_{\mathbf{k}} \Omega_{2}$. The corresponding Lagrangian is thus given by

$$
L_{\mathrm{mf}}=\frac{1}{2} \sum_{\mathbf{k}} \Psi^{\dagger}\left(\mathbf{k}, i \omega_{n}\right)\left[i \omega_{n} \Omega_{1}-H_{\mathrm{mf}}(\mathbf{k})\right] \Psi\left(\mathbf{k}, i \omega_{n}\right)
$$

Here the generalized $8 \times 8$ Dirac matrices have been defined by $\Omega_{1}=\sigma_{z} \otimes \sigma_{0} \otimes \sigma_{0}, \Omega_{2}=\sigma_{x} \otimes \sigma_{z} \otimes \sigma_{z}$, where $\sigma_{x}, \sigma_{y}, \sigma_{z}$, and $\sigma_{0}$ are three Pauli $2 \times 2$ matrices and a unity matrix, respectively. $\Omega_{0}$ is the $8 \times 8$ unity matrix, and $\Omega_{1}$ and $\Omega_{2}$ obey the anticommutation relation $\left[\Omega_{1}, \Omega_{2}\right]_{+}=2 \Omega_{0}$. From the Lagrangian, the bosonic Matsubara Green function is given by

$$
G\left(\mathbf{k}, i \omega_{n}\right)=\frac{-i \omega_{n} \Omega_{1}-\lambda \Omega_{0}+2 Z \Delta \gamma_{\mathbf{k}} \Omega_{2}}{\omega_{n}^{2}+\left[\lambda^{2}-\left(2 Z \Delta \gamma_{\mathbf{k}}\right)^{2}\right]},
$$

which implies that the bosonic quasiparticle excitations form a continuum band, and their dispersion relation is $\omega_{\mathbf{k}}=\sqrt{\lambda^{2}-\left(2 Z \Delta \gamma_{\mathbf{k}}\right)^{2}}$. Moreover, the free energy can be evaluated as

$$
F_{\mathrm{mf}}=\frac{1}{\beta} \sum_{\mathbf{k}, \mu} \ln \left[2 \sinh \left(\frac{\beta \omega_{\mathbf{k}}}{2}\right)\right]+Z \Delta^{2} N-3 \lambda N,
$$

and the saddle point equations are derived as follows:

$$
\begin{aligned}
& \frac{1}{N} \sum_{\mathbf{k}} \frac{2 \lambda}{\sqrt{\lambda^{2}-\left(2 Z \Delta \gamma_{\mathbf{k}}\right)^{2}}}\left[2 n_{B}\left(\omega_{\mathbf{k}}\right)+1\right]=3, \\
& \frac{1}{N} \sum_{\mathbf{k}} \frac{\left(2 Z \gamma_{\mathbf{k}}\right)^{2}}{\sqrt{\lambda^{2}-\left(2 Z \Delta \gamma_{\mathbf{k}}\right)^{2}}}\left[2 n_{B}\left(\omega_{\mathbf{k}}\right)+1\right]=Z .
\end{aligned}
$$

The first equation corresponds to the request of the local constraint on average, and the second one determines the SU(4) short-ranged VB order parameter self-consistently.

To get the collective excitations of the model, the dynamical correlation functions of the spin, orbital, and combined spin-orbital density operators should be calculated. The corresponding density operators can be written in terms of the Nambu spinor introduced above,

$$
\begin{aligned}
S_{i}^{z} & =\frac{1}{4} \Psi^{\dagger}\left(\mathbf{r}_{i}\right) \Omega_{3} \Psi\left(\mathbf{r}_{i}\right), \\
T_{i}^{z} & =\frac{1}{4} \Psi^{\dagger}\left(\mathbf{r}_{i}\right) \Omega_{4} \Psi\left(\mathbf{r}_{i}\right), \\
L_{i}^{z z} & =\frac{1}{4} \Psi^{\dagger}\left(\mathbf{r}_{i}\right) \Omega_{5} \Psi\left(\mathbf{r}_{i}\right),
\end{aligned}
$$

where $\Omega_{3}=\sigma_{z} \otimes \sigma_{0} \otimes \sigma_{z}, \Omega_{4}=\sigma_{z} \otimes \sigma_{z} \otimes \sigma_{0}$, and $\Omega_{5}=\sigma_{0} \otimes \sigma_{z} \otimes \sigma_{z}$ are introduced. Except for the anticommutation relations $\left[\Omega_{1}, \Omega_{2}\right]_{+}=\left[\Omega_{2}, \Omega_{3}\right]_{+}=$ $\left[\Omega_{2}, \Omega_{4}\right]_{+}=2 \Omega_{0}$, all other relations between the Dirac matrices from $\Omega_{1}$ to $\Omega_{5}$ satisfy the commutation relations.

In a $1 \mathrm{D}$ system, we have $Z=2$ and $\gamma_{k}=\cos k$. At $T=0 \mathrm{~K}$, there are no particle excitations $n_{B}\left(\omega_{k}\right)=0$. When the summations over momenta are converted into the integrals within the first Brillouin zone, the self-consistent equations are easily solved, leading to $\Delta \simeq 1.67476$ and $\lambda \simeq 7.31391$. The corresponding ground state energy per site is given by $\varepsilon_{g}=-2 \Delta^{2} \simeq-5.60964$, and there is a finite gap in the bosonic quasiparticle excitation spectrum, $\omega_{0}=\sqrt{\lambda^{2}-16 \Delta^{2}} \simeq 2.93533$ at the momentum $k=0, \pi$. These results have shown that the ground state is a quantum disordered state of resonating valence bonds, in qualitative agreement with the previous numerical calculations [16]. Moreover, the dynamical susceptibilities at zero temperature can be easily evaluated as follows:

$$
\begin{aligned}
\chi(q, \omega+i \eta)= & \int \frac{d k}{16 \pi}\left[\frac{\lambda^{2} \pm 16 \Delta^{2} \cos k \cos (k+q)}{\omega_{k} \omega_{k+q}}-1\right] \\
& \times\left[\frac{1}{\omega+\omega_{k}+\omega_{k+q}+i \eta}-\frac{1}{\omega-\omega_{k}-\omega_{k+q}+i \eta}\right],
\end{aligned}
$$

where $\eta$ is a positive infinitesimal, both the spin and orbital dynamic susceptibilities are equal to each other and choose the lower minus sign, and the combined spinorbital dynamic susceptibility takes the upper plus sign. The dynamic density spectra are then easily obtained:

$$
\begin{aligned}
\operatorname{Im}_{\chi_{S}}(q \rightarrow Q, \omega) & =\operatorname{Im} \chi_{T}(q \rightarrow Q, \omega) \\
& =\operatorname{Im} \chi_{L}(q \rightarrow 0, \omega) \\
& =\frac{\pi}{16|\omega|} \sqrt{\frac{4 \lambda^{2}-\omega^{2}}{\omega^{2}-4 \lambda^{2}+64 \Delta^{2}}},
\end{aligned}
$$

where $2 \omega_{0}<|\omega|<2 \lambda$. Clearly, there is a finite energy gap $E_{r}=2 \omega_{0} \simeq 5.87066$ in both the spin and or- bital collective excitations at $q=Q$, and in the combined spin-orbital collective excitation at $q=0$. Therefore we conclude that the ground state is a quantum disordered spin-orbital liquid state with a finite-energy gap in the collective excitations.

On a $2 D$ square lattice, $Z=4$ and $\gamma_{\mathbf{k}}=\frac{1}{2}\left(\cos k_{x}+\right.$ $\cos k_{y}$ ). At zero temperature, the conversion from the summations over momenta to the integrals will be invalid as $(8 \Delta) \rightarrow \lambda$. Following the analogous treatments for the Bose-Einstein condensation [22], we separate the divergent terms at $\mathbf{k}^{*}=\mathbf{0}$ and $\mathbf{k}^{*}=\mathbf{Q}$ from the summations to yield 


$$
\begin{aligned}
& \int \frac{d^{2} k}{(2 \pi)^{2}} \frac{\lambda}{\sqrt{\lambda^{2}-\left(8 \Delta \gamma_{\mathbf{k}}\right)^{2}}}+\frac{4 n_{B}\left(\omega_{\mathbf{k}^{*}}\right)}{\sqrt{1-(8 \Delta / \lambda)^{2}}}=\frac{3}{2}, \\
& \int \frac{d^{2} k}{(2 \pi)^{2}} \frac{\lambda \gamma_{\mathbf{k}}^{2}}{\sqrt{\lambda^{2}-\left(8 \Delta \gamma_{\mathbf{k}}\right)^{2}}}+\frac{4 n_{B}\left(\omega_{\mathbf{k}^{*}}\right)}{\sqrt{1-(8 \Delta / \lambda)^{2}}}=\frac{\lambda}{16} .
\end{aligned}
$$

When $(8 \Delta) \rightarrow \lambda$, the boson condensation occurs, and the ratio $\rho=4 n_{B}\left(\omega_{\mathbf{k}^{*}}\right) / \sqrt{1-(8 \Delta / \lambda)^{2}}$ is finite, which can be determined from the first equation by setting $\lambda=8 \Delta$ inside the integral: $\rho \simeq 0.107$. Similarly, from the second equation, one can also obtain the order parameter $\Delta \simeq$ 1.3159 and thus $\lambda \simeq 10.5271$. Moreover, the ground state energy per site is evaluated to be $\varepsilon_{g}=-4 \Delta^{2} \simeq-6.9263$. The bosonic quasiparticle excitation spectrum now becomes linearly dependent on momentum near the minimal points $\mathbf{k}^{*}=\mathbf{0}, \mathbf{Q}$. When the boson condensation is carefully treated, the dynamical structure factors for spin, orbital, and combined spin-orbital collective excitations can also be calculated through the fluctuation dissipation theorem. After some algebra, the final results are given by

$$
\begin{aligned}
S_{S}(\mathbf{q} \rightarrow \mathbf{Q}, \omega) & =S_{T}(\mathbf{q} \rightarrow \mathbf{Q}, \omega)=S_{L}(\mathbf{q} \rightarrow 0, \omega) \\
& =\frac{\pi \rho^{2}}{2} \delta(\omega) \operatorname{sgn} \omega \ldots,
\end{aligned}
$$

where $K(x)$ is the complete elliptical function of the second kind. In the range of $0<|\omega|<2 \lambda$, there is a sharp resonance in the collective excitations at zero frequency, corresponding to the formation of a novel long-range ordered spin-orbital crystal state of resonating valence bonds. In such a state, both the spin and orbital degrees of freedom from an AFM long-range order separately, while the combined spin-orbital degrees of freedom form an FM long-range ordering. The corresponding magnetizations can be read off from the coefficient of the delta function $m=\rho / 2 \simeq 0.0535$, which is much smaller than that of the $\mathrm{SU}(2)$ antiferromagnetic Heisenberg spin model. Actually, this result is consistent with the identity in Eq. (3), as the VB ordered state in the mean field theory has kept the SU(4) symmetry of the model. The present results are in conflict with the prediction of a spin-liquid state with a finite-energy gap by the quantum Monte Carlo Green function calculations on a small square lattice for the same model [17]. However, the very small magnetization clearly indicates that the quantum fluctuations in the spin-orbital coupled model are much stronger than those in the pure spin model. The numerical data show that the energy gap (even if it exists) is very close to zero. In this sense, both analytical and numerical results seem to be consistent with each other. Compared with the numerical method, the SU(4) Schwinger boson mean field approach for the sym- metrically coupled spin-orbital systems has provided the insight into the exact nature of the ground state and its first excitations.

In conclusion, we derived a reduced effective spinorbital Hamiltonian in a symmetric spin-orbital system by introducing the SU(4) Schwinger boson representation. After introducing a symmetric pairing VB order parameter, a mean field theory was developed, leading to a quantum disordered liquid state with a finite gap in the collective excitations in 1D and, on a 2D square lattice, a novel long-range FM ordered spin-orbital crystal state, where both the spin and orbital degrees of freedom form AFM. The new ordering properties due to the interplay between the spin and orbital degrees of freedom should be detected in future experiments on transitional metal oxides.

This work was supported by a RGC grant of Hong Kong. G.-M. Zhang thanks Professor Lu Yu for stimulating his interest in this subject, and acknowledges the partial support from NSF-China (Grant No. 10074036) and the Special Fund for Major State Basic Research Projects of China (G2000067107).

[1] W. Bao et al., Phys. Rev. Lett. 78, 507 (1997); C. Broholm et al., J. Appl. Phys. 79, 5023 (1996).

[2] T. Mizokawa et al., Phys. Rev. B 61, R3776 (2000).

[3] G. Khaliullin and S. Maeckawa, Phys. Rev. Lett. 85, 3950 (2000).

[4] E. Saitoh et al., Nature (London) 410, 180 (2001).

[5] K. I. Kugel and D. I. Khomskii, Usp. Fiz. Nauk 136, 621 (1982) [Sov. Phys. Usp. 25, 231 (1982)].

[6] L. F. Feiner et al., Phys. Rev. Lett. 78, 2799 (1997).

[7] D. P. Arovas and A. Auerbach, Phys. Rev. B 52, 10114 (1995).

[8] S. K. Pati and R. R. P. Singh, Phys. Rev. Lett. 81, 5406 (1998).

[9] J. van den Brink et al., Phys. Rev. B 58, 10276 (1998).

[10] Y. Q. Li et al., Phys. Rev. Lett. 81, 3527 (1998).

[11] B. Frischmuth et al., Phys. Rev. Lett. 82, 835 (1998).

[12] A. Joshi et al., Phys. Rev. B 60, 6584 (1999).

[13] P. Azaria et al., Phys. Rev. Lett. 83, 624 (1999).

[14] Y. Yamashita et al., J. Phys. Soc. Jpn. 69, 242 (2000).

[15] C. Itoi et al., Phys. Rev. B 61, 6747 (2000).

[16] G. Santoro et al., Phys. Rev. Lett. 74, 4039 (1995); Phys. Rev. B 53, 828 (1996); ibid. 55, 16168 (1997).

[17] G. Santoro et al., Phys. Rev. Lett. 83, 3065 (1999).

[18] P. Paul et al., J. Am. Chem. Soc. 116, 4145 (1994).

[19] J. Choi et al., Phys. Rev. Lett. 80, 1328 (1998); J. Choi et al., Phys. Rev. B 59, 1819 (1999).

[20] D. P. Arovas and A. Auerbach, Phys. Rev. B 38, 316 (1988); A. Auerbach and D. P. Arovas, Phys. Rev. Lett. 61, 617 (1988).

[21] J. E. Hirsch and S. Tang, Phys. Rev. B 39, 2850 (1989).

[22] See, for example, K. Huang, Statistical Mechanics (McGraw-Hill, New York, 1956). 